\title{
'n Toetskriterium vir kategorieel kompakte ringe
}

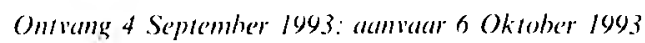

ABSIRACT

A test criterion for categorically compact rings

The Karatosski-Mrowka theorem - a topological space $X$ is compact if and only if for each space Y the second projection map $\pi_{2}: X \times Y \rightarrow Y$ is a dosed map - provides for a categorical interpretation of compactness once a suitable notion of closure is ohtained. In this article we study a general notion of compactness with respect to a class , F of rings that is either hereditary semisimple or quotient reflective. We show that to test for categorical compactmess of a ring $R$ we need only check $R$ R, $\bar{F}$, where $R \bar{F}=\bigcap\{D \quad D$ is an ideal of $R$ and $R, D \in \mathscr{F}\}$, and to test $\pi_{2}:(R / R, \bar{F}) \times H \rightarrow H$ for rings $H$ helonging $t o$. $\vec{F}$ as opposed to arhitrary $H$.

Eicnskappe en verbande tussen die topologiese begrippe "kompak" en "Hausdorff" kan meer abstrak bestudeer word, met behoud van eienskappe en verbande. Iie Kuratowski-Mrówka stelling maak dit moontlik om kompaktheid in kategorieë te bestudeer nadat "n geskikte geslotenheidsbegrip uitgevoer is. 1.2 .3 .4 .5 .6 Dit word via kategoriële afsluitoperatore bereik. ${ }^{4.6}$ Die klas van kategorieel kompakte objekte (met betrekking tot in afsluitoperator) is geslote onder die neem van beelde en eindige produkte. Vir sekere afsluitoperatore het die klassieke resultate dat geslote deelruimtes van kompakte ruimtes kompak is en dat kompakte deelruimtes van Hausdorff-ruimtes geslote is, analogiee in kategorieë.

In kategorieë van module is daar 'n verband tussen kategoriële kompaktheid met betrekking tot 'n afsluitoperator en relatiewe deelbaarheid. Analogieë van die gemelde klassieke resultate geld. ${ }^{5.6 .7}$ Torsievrye klasse kan gebruik word om geslotenheid in verskeie kategorice van groepe te definicer ("n ondergroep $A$ van 'n groep $G$ word "geslote" genoem as dit 'n normaaldeler is en $G_{l} A$ torsievry is). In sulke gevalle is daar 'n verband tussen katogoriële kompaktheid en die bestaan van $n$-de magswortels. ${ }^{8}$ In kategorieë van Abelse grocpe en nilpotente groepe is kategoriële kompaktheid met betrekking tot gereduseerde groepe presies dic kotorsiegroepe."

Die gemelde resultate toon dat kategorieel kompakte objekte interessante eienskappe het en dat sommige belangrike klasse van objekte kategorieel kompak is.

In hierdie aankondiging word kompaktheid met betrekking tot 'n klas $\bar{F}$ van ringe beskou, waar $\bar{F}$ of halfeenvoudig-erflik of kwosiëntreflektief is. Ons toon aan dat vir 'n ring $R$ om kategorieel kompak te wees, dit voldoende is om $R$ R. $\bar{F}$ te beskou wat $R \cdot \bar{F}=\bigcap\{D: D$ 'n ideaal in $R$ en $R / D \in \tilde{F}$, en dat dit ook voldoende is om $\pi_{2}: R / \tilde{H} \times H \rightarrow H$ te ondersoek vir $H \in \mathscr{F}$. Hoewel die resultate vir ringe geformuleer word, kan dit ook in ander kategorieë as toetsprosedure vir kategoriële kompaktheid gebruik word.

Deel (iii) van die volgende definisie is geïnspireer deur die werk van Dikranjan en Guili:
Definisie: Laat ./l en 1 isomorfgestote klasse van thie nooduendig assosiatiewe) ringe wees wat o beval, (en $R$ in ring.

(i) $R$ heet in. ll-gestole deelring v'an $Q$ as $R \triangleleft Q \mid R$ 'n ideaal in $Q$ ) is en $Q R \in$. $/$.

(ii) $R$ heeet . /l-Hatusdorff as $R \in$. $/$.

(iii) $R$ heet (. II; 1)-kompak as vir elke ring $I /$ e'n elke . IIgeslote declring $A$ l'an $R \times I I$ geld dat $\pi_{2}(A)$ 'n . l' geslote deelring rom $I I$ is naar $\pi_{2}: R \times I I \rightarrow I I$ die meede projeksie is. Kortweg word (. I, . /l)-kompak . IIkompak genoem.

Die begrip kategorieel kompak is eerste deur Manes' bestudeer en later deur Herrlich, Salicrup en Strecker. ${ }^{3}$ Castellini ${ }^{4}$ het dié begrip via 'n afsluitoperator ontwikkel. Die volgende resultate geld vir ringe:

Proposisie Laat . II, I en " willekearige klasse wees.

(i) As $R_{1}\left(. / /, 1\right.$, )-kompak is en $R_{2}$ is (.1. ( ) ) -kompak dan is $R_{1} \times R_{2}(. / 1:($ C) -kompak.

(ii) Homomorfe beelde lan $\left(. / 1,1^{\prime}\right)$-kompakte ringe is $(. \| 1 ;-\mathcal{A})-k o m p a k$.

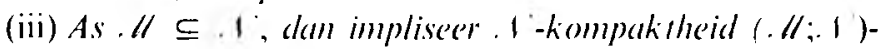
kompaktheid.

Stelling 1 Laat. II en 1 klasse wees, $R$ in ring en heskou die bewerings:

(i) $R$ is $(.11,1.1)-k o m p a k$.

(ii) $R / R \cdot \|$ is $(. \| ; \cdot$. $)$-kompak waar R./l $=\cap\{D \triangleleft R: R /$ $D \in \bar{F}\}$.

(iii) $R / D$ is $(. \| ;, V)$-kompak vir elke . It-geslote deelring $D$ van $R$.

Dan geld:

I Vir willekerrige./l en, 1, (i) $\Longrightarrow$ (ii) $\Longrightarrow$ (iii).

II As. It erflik is (geslote onder die neem van ideale), dam (iii) $\Longrightarrow$ (i) en dus

(i) $\Longleftrightarrow$ (ii) $\Longleftrightarrow$ (iii).

III As . II geslote is onder subdirekte produkte, dain (iii) $=$ (ii) en dus

(ii) $\Longleftrightarrow$ (iii). 
in Radikaalpaar ( $\mathscr{R}: \mathscr{F})$ is 'n par van klasse met $\mathscr{F}$ ' halfeenvoudige klas en $\mathscr{A}$ die korresponderende radikaalklas. 'n Torsicteoric vir ringe is 'n radikalalpaar $(\bar{T}: \overline{\mathcal{H}})$ met $\bar{F}$ erflik. 'n Erflike halfeenvoudige klas word in torsievrye klas genoem en die korresponderende radikaalklas heet 'n torsieklas." Die volgende stelling toon hoe die toets vir kompaktheid vereenvoudig kan word deur slegs sekere ringe $H$ te beskou. Dit veralgemeen 'n resultaat vir kwosiëntreflek tiewe klasse van groepe. ${ }^{11}$

Stelling 2 Laat $\bar{\not}$ in klas wees wat of torsievry of kwosiëntreflektief' is en 's 'n willekeurige klas. 'n Ring $R$ is $(\bar{F}:(G)$-kompak as en slegs as vir elke $I I \in, \bar{F}$ en vir elke $\bar{F}$-geslote deelring $A$ van $R \times \| l$ geld dat $\pi_{2}(A)$ Geslote is in $I I$ watar $\pi_{2}: R \times I t \rightarrow I I$ die tweede projeksie is.

Bewys Die nodigheid is triviaal. Laat $\overline{\mathcal{F}}$ 'n torsievrye klas wees. (Die bewys vir die geval waar $\tilde{\mathcal{F}}$ kwosiëntreflektief is verloop soortgelyk, want volgens Stelling I kan aangeneem word dat $R \in \tilde{F}$ ). Laat $I I$ willekeurig wees en $A$ 'n $\mathscr{F}$-geslote deelring van $R \times H$. Laat $\mathscr{T}$ die radikaalklas wees bepaal deur $\mathscr{F}$ en laat $\mathscr{T} H=\sum\{D \quad D \triangleleft$ $H$ en $D \in \tilde{T}\}$. Dan is $\tilde{T} H \in \tilde{T}$. Laat $\eta: H \rightarrow H / \bar{T} H$ en $\eta^{\prime}: R \times H \rightarrow \frac{R \times H}{A}$ die natuurlike afbeeldings wees. Laat $B$ die beeld van $A$ in $R \times(H / T H)$ wees en $\frac{R \times(H \cdot T H)}{B}=\mathrm{Q}$ Omdat. $\bar{T}$ 'n torsieklas is, is dit geslote onder homomorfe beelde. Gevolglik is $\eta^{\prime}(0 \times, \bar{T} H) \in \mathscr{T}$. Omdat $\eta^{\prime}(0 \times \mathscr{T} H) \triangleleft \frac{R \times H}{A}$, en $\mathscr{F}$ erflik is, volg $\eta^{\prime}(0 \times \mathscr{T} H) \in \mathscr{F}$. Aangesien $\overline{\mathscr{F}} \cap \bar{A}=0$ is $0 \times \bar{T} H \subseteq A$ en dus is $\frac{R \times H}{A} \cong Q$ en gevolglik is $B$ 'n $\tilde{\mathscr{F}}$-geslote deelring van $R \times(H / \mathscr{T} H)$. Omdat $(H / \tilde{T} H) \in \mathscr{F}$ volg uit die aanname dat $\frac{(H, \pi H)}{\pi_{2} B} \in \mathscr{G}$. Laat $A_{2}$ en $B_{2}$ die beclde van $A$ en $B$ onder $\pi_{2}$, aandui. Ons het bewys dat $\pi H \subseteq A_{2}$, dus $\bar{T} H \cap A_{2}=\tilde{T} H$. Gevolglik is $H_{/} A_{2} \cong \frac{(H(T H H)}{B_{2}}$ en $A_{2}$ is $\mathscr{G}$-geslote in $H$

Voorbeeld ${ }^{8}$ Daar is $n$ interessante situasie vir die klas van (gewone) torsievrye ringe. ${ }^{8.12} \mathrm{Vir}$ elke ring $R$ vorm die (gewone) torsie-elemente $\tau R$ 'n ideaal met $R / \tau R$ torsievry. Laal $\tilde{F}_{T}$ die klas van alle torsievrye ringe aandui. Dan is $\overline{\mathcal{F}}_{t}$ die torsievrye klas van 'n erflike torsieteorie in die kategorie van nienoodwendige assosiatiewe ringe. Laat $R^{\prime}$ die Abelse groep van die ring $R$ aandui. As $R^{\prime} / \tau R^{\prime}$ (opgeval as Abelse groep) deelbaar is dan is $R \mathscr{F}_{\tau^{-}}$ kompak. Gevolglik is elke Artin-ring en elke reguliere ring $\mathscr{F}_{\tau}$-kompak. As $R$ 'n nulring is dan is $R \tilde{F}_{\tau}$-kompak as en slegs as $R^{\prime} / \tau R^{\prime}$ (opgevat as Abelse groep) deelbaar is. Nie alle ringe is daarom $\mathscr{F}_{\tau}$-kompak nie. Elke ring met of 'n linker of ' $n$ regter identiteit is $\mathscr{F}_{\tau}$-kompak.

Laat $\mathbb{Z}^{0}$ die nulring op die heelgetalle aandui en laat
$\mathbb{Z}^{1}$ die ring wees wat ontstaan deur aan $\mathbb{Z}^{(0)}$ ' identeit toe te voeg. Dan is $\mathbb{Z}^{0}$ ' $\mathrm{n}$ ideaal van $\mathbb{Z}^{1}$ en $\mathbb{Z}^{1} / \mathbb{Z}^{0} \cong \mathbb{Z}$. Gevolglik

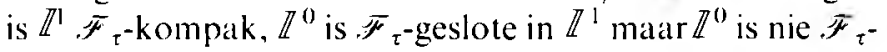
kompak.

As ring is $n \mathbb{Z}, \tilde{F}_{\tau}$-kompak en 'n ideaal van $\mathbb{Z}$. Die ring $\mathbb{Z}$ behoort tot $\bar{F}_{\tau}$ en is dus $\bar{F}_{\tau}$-Hausdorff, maar $\mathbb{Z} / \mathrm{n} \mathbb{Z}$ is torsie en daarom is $\mathrm{n} \mathbb{Z}$ nie $\tilde{\mathscr{F}}_{\tau}$-geslote in $\mathbb{Z}$.

Die klassieke resultate genoem in die inleiding geld dus nie vir die klas $\mathscr{F}_{\tau}$ nie.

Die kriterium wat hier bespreek is, maak dit aansienlik makliker om vas te stel watter objekte kategorieel kompak is. Implikasies hiervan word tans ondersoek.

\section{T.H. FAY}

Technikon Pretoria en Universiteit van Suidelike Mississippi, VSA

\section{S.V. JOUBERT*}

Departement Kwaliteitsversekering en Statistiek, Technikon Pretoria, Privaat sak x680, Pretoria 0001

\section{M.J. SCHOEMAN}

Departement Wiskunde en Toegepaste Wiskunde, Universiteit van Pretoria, Pretoria 0002

* Outeur aan wie korrespondensie gerig kan word.

\section{ERKENNING}

Die Departement Kwaliteitsversekering en Statistiek, Technikon Pretoria waar hierdie werk onderneem is, word bedank, asook die SNO vir finansiële ondersteuning.

\section{IITERATUURVERWYSINGS}

1. Manes, E.G. (1979). Compact Hausdorlf Objects, Topolog! and its Applicarions. 4. 341-360.

2. Cassidy, C.. Hebert, M. \& Kelly, G.M. (1985). Reflective subcategorics, localizations and factorization systems, J. Austral. Math. Soc. (Series A), 38, 287-329.

3. Herrlich, H., Salicrup. G. \& Strecker, (j.E. (1987). Factorizations, denseness, separation, and relatively compact objects, Topology and its Applications. 27, 157-169.

4. Castellini, G. (1990). Compact objects, surjectivity of epimorphisms and compactifications, Cahiers de Topologie et Géométrie Différentielle Cakegoriques, $\mathrm{XXX1-1,53-65.}$

5. Fay, T.H. (1988). Compact modules, Comm. Algebra, 16, 1209-1219.

6. Dikranjan, D. \& Giuli, E. (1991). Factorizations, injectivity and Compactness in categories of modules. Comm. Algehra. 19. 45-83.

7. Fay, T.H. \& Joubert, S.V. Relative injectivity. Chinese Journal of Mathematics (in press).

8. Fay. T.H. \& Joubert, S.V. Categorically compact rings (presented).

9. Fay, T.H. \& Walls. G.L. The Mal'cev completion, the p-cotorsion completion, and categorical compactness for nilpotent groups (presented).

10. Leavitt, W.G. \& Wiegandt, R. (1979). Torsion theory for not necessarily associative rings, Rocky Mountain J. Math., 9, 259-271.

11. Fay T.H. \& Schoeman, M.J., The $\mathbb{Z}$-adic completion, the p-adic completion, and categorical compactness for abelian and nilpotent groups (in preparation).

12. Joubert, S.V. (1992). Categorically compact rings and modules, Chinese J. Math., 20, 247-365. 